\title{
Human papillomavirus viral load as a useful triage tool for non-16/18 high-risk human papillomavirus positive women: A prospective screening cohort study
}

\author{
Li Dong a,b,1, Margaret Z. Wang c,d,1, Xue-lian Zhao a , Rui-mei Feng a , Shang-ying Hu ${ }^{\text {a }}$, Qian Zhang a , \\ Jennifer S. Smith ${ }^{\text {e,f }}$, You-lin Qiao a , Fang-hui Zhao ${ }^{\text {a,* }}$ \\ a Department of Epidemiology, National Cancer Center/Cancer Hospital, Chinese Academy of Medical Sciences (CAMS) and Peking Union Medical College (PUMC), Beijing, China \\ b Institutes of Biomedical Sciences, Shanxi University, Taiyuan, China \\ ' Pritzker School of Medicine, University of Chicago, Chicago, IL 60637, USA \\ d UJMT Fogarty Consortium, NIH Fogarty International Center, Bethesda, MD, USA \\ e Department of Epidemiology, Gillings School of Global Public Health, University of North Carolina, Chapel Hill, NC, USA \\ ${ }^{\mathrm{f}}$ UNC Lineberger Comprehensive Cancer Center, University of North Carolina, Chapel Hill, NC, USA
}

\section{H I G H L I G H T S}

- First comparison of risk triage of type-specific HPV by viral load, cytology or VIA

- HPV16/18 infection was at high risk, even with normal cytology or low viral load.

- Triage non-16/18 HPV infection by viral load is viable when cytology is unavailable.

\section{A R T I C L E I N F O}

Article history:

Received 15 August 2017

Received in revised form 6 November 2017

Accepted 10 November 2017

Available online 21 November 2017

\section{Keywords:}

Human papillomavirus

Viral load

Genotypes

Cervical cancer

Risk stratification

Triage

\begin{abstract}
A B S T R A C T
Objective. ASCCP cervical cancer screening guidelines recommend triaging high-risk human papillomavirus (hrHPV) positive women with cytology and genotyping, but cytology is often unavailable in resource-limited areas. We compared the long-term risk of cervical cancer and precancers among type-specific hrHPV-positive women triaged by viral load to cytology and visual inspection with acetic acid (VIA).

Methods. A cohort of 1742 Chinese women was screened with cytology, VIA, and Hybrid Capture 2 (HC2) test and followed for ten years. All HC2-positive samples were genotyped. Viral load was measured by HC2 relative light units/cutoff (RLU/CO). Ten-year cumulative incidence rate (CIR) of cervical intraepithelial neoplasia grade 2 or worse (CIN2 +) for type-specific hrHPV viral load was estimated using Kaplan-Meier methods.

Results. Baseline hrHPV viral load stratified by specific genotypes was positively correlated with prevalent cytological lesions. Ten-year CIR of CIN2 + was associated with cytological lesions and viral load. Among HPV 16/ 18-positive women, ten-year CIR of CIN2 + was high, even with normal cytology (15.3\%), normal VIA (32.4\%), viral load with $\mathrm{RLU} / \mathrm{CO}<10$ (23.6\%) or RLU/CO $<100$ (33.8\%). Among non-16/18 hrHPV positive women, tenyear CIR of CIN2 + was significantly stratified by cytology grade of atypical squamous cell of undetermined significance or higher (2.0\% VS. 34.6\%), viral load cutoffs at $10 \mathrm{RLU} / \mathrm{CO}$ (5.1\% VS. $27.2 \%)$, at $100 \mathrm{RLU} / \mathrm{CO}$ (11.0\% VS. $35.5 \%)$, but not by VIA (19.1\% VS. $19.0 \%$ ).

Conclusions. Our findings support the guidelines in referring all HPV16/18 positive women to colposcopy and suggest triaging non-16/18 hrHPV positive women using viral loads in resource-limited areas where cytology screening was inaccessible.
\end{abstract}

(c) 2017 Elsevier Inc. All rights reserved.

Abbreviations: HPV, Human papillomavirus; hr, High risk; CIN, Cervical intraepithelial neoplasia; NILM, Negative for intraepithelial lesion or malignancy; ASCUS, Atypical squamous

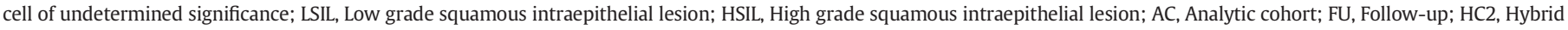
Capture 2; LBC, Liquid-based cytology; VIA, Visual inspection with acetic acid; CIR, Cumulative incidence rate.

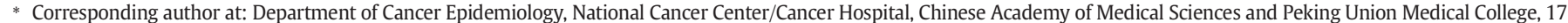
South Panjiayuan Lane, P.O. Box 2258, Beijing 100021, China.

E-mail address: zhaofangh@cicams.ac.cn (F. Zhao).

1 Contributed equally to this article. 


\section{Introduction}

The well-recognized knowledge that high-risk human papillomavirus (hrHPV) is a necessary etiology for progression to cervical cancer is reinforcing HPV DNA testing incorporated into cervical cancer screening programs in many countries [1-3]. But potential risk of excessive colposcopy and overtreatment would occur in women with transient HPV infections who regressed spontaneously within one to two years $[4,5]$. Therefore how to best triage HPV-positive women through secondary screening to identify those women with true precancerous lesions remains a pending issue in cervical cancer screening.

Given the different carcinogenicity of HPV among genotypes, partial typing tests such as HPV 16/18 and HPV 16/18/45 are being integrated into cervical screening to improve the risk stratification of general population [6-8]. In addition, other triage techniques are also desirable. The updated guidelines released by American Society for Colposcopy and Cervical Pathology (ASCCP) recommended the cytology for triaging the non-16/18 hrHPV positive women [9]. It is no doubt of high specificity of cytology screening [10], nevertheless, high-quality cytology screening programs are often unavailable due to the lacking of trained cytopathologists, limited healthcare resources, or poor infrastructures in low-resource countries. VIA is another option for secondary screening of HPV-positive women in low and middle income settings but with a wide variation of sensitivity rate from $41 \%$ to $92 \%$ for CIN2 + detection between providers [11].

Viral load resulting from productive viral replication might predict the risk of viral persistence and the progression to high-grade CIN and cervical cancer [12-16]. The likelihood of the viral load related risk was reported to be dependent on specific HPV genotypes, as demonstrated by many large screening population studies from US, Belgium and Denmark [17-20]. However, few studies focused on the longitudinal comparison of risk stratification of HPV viral load in comparison to cytology or VIA to date.

Our previously prospective study of a six-year follow-up cervical cancer screening cohort of 1997 women demonstrated that baseline high hrHPV viral load was associated with the increased risk of progression to CIN2 + and potentially served as a biomarker to triage hrHPV-positive women for colposcopy [21], however, did not specify the risk of CIN2 + by individual hrHPV type. In this present study, with the data of a ten-year follow-up of the same cohort, we further compared the long-term risk stratification of CIN2 + by hrHPV viral loads against cytology and VIA among HPV 16/18 and non-16/18 hrHPV-positive women, so as to investigate the feasibility of viral load as an alternative triage method for hrHPV-positive women in lieu of cytology, especially in regions with high-quality cytology examination unavailable.

\section{Methods}

\subsection{Study population}

A cohort of 1997 women, aged 35-45 years., married, not pregnant, and without history of hysterectomies, were enrolled in Shanxi Province of Cervical Cancer Screening I (SPOCCS I) study in 1999 [22]. These participants were then followed up through three organized visits in 2005 , 2010 , and 2014, respectively $[23,24]$. The incidence and mortality of cervical cancer in this cohort were also recorded by a national cancer registry. This study was approved by the Institutional Review Board of Cancer Institute/Hospital, Chinese Academy of Medical Sciences (CICAMS).

\subsection{Clinical examinations}

Each participant was screened with liquid-based cytology (LBC), Hybrid Capture 2 (HC2) testing, visual inspection with acetic acid (VIA) in
1999, 2005, 2010, and 2014 (except for VIA in 2014). Women positive by any of three tests were referred for colposcopy, and lesions visible under colposcopy were directly biopsied. Cytology results were interpreted using the Bethesda classification system and histological diagnoses were made according to CIN classifications. Histological diagnoses of CIN grade 2, CIN grade 3, squamous cell carcinoma (SCC), and adenocarcinoma in situ (AIS), or adenocarcinoma were categorized as CIN2 +. Women with histology-confirmed CIN2 + lesions were recommended for treatment as per local clinical guidelines.

\subsection{HPV DNA testing}

HC2 assay was conducted on cervical cytological samples to test the presence of HPV DNA within two weeks of specimen collection. This assay detects the DNA of 13 carcinogenic hrHPV types (HPV 16, 18, 31, $33,35,39,45,51,52,56,58,59$ and 68 ) but unable to discriminate individual genotypes. Samples were deemed as hrHPV-positive if the signal strength in relative light units compared with standard positive control (RLU/CO) in HC2 assay was $1.0(1 \mathrm{pg} / \mathrm{mL}$, approximately 5000 viral copies) or higher. A semi-quantitative viral load of women positive for HPV were then categorized into three groups: low (1.0-9.9 RLU/CO), moderate (10.0-99.9 RLU/CO) and high viral load ( $\geq 100.0 \mathrm{RLU} / \mathrm{CO}$ ), with the same criteria made by our previous study and other studies $[12,21,25,26]$.

\subsection{HPV genotyping}

All HC2-positive cytological specimens were genotyped using PCRbased reverse hybridization line probe assay (INNO-LiPA Extra, Innogenetics, Belgium) with a $\mathrm{SPF}_{10}$ primers set (DDL diagnostic laboratory, Netherlands) ( $\mathrm{SPF}_{10}$-LiPA). This assay identifys $28 \mathrm{hrHPV}$ types on a line strip, including 13 hrHPV types covered by the HC2 assay, three probable carcinogenic genotypes (26, 53, and 66), and 12 low-risk genotypes $(6,11,40,43,44,54,69,70,71,73,74$, and 82$)$. We defined hrHPV infections as any positive indication of HPV 16, 18, 31, 33, 35, $39,45,51,52,56,58,59$ or 68 . Specimens were deemed as hrHPV-negative if $\mathrm{HC2}$ assay result was negative or if $\mathrm{SPF}_{10}$-LiPA assay result for these 13 hrHPV genotypes was negative.

\subsection{Statistical analysis}

A total of 1742 women were examined in 2005 after excluding 255 women due to loss to follow up, hysterectomy, or death during 19992005. Thereafter, 209 hrHPV-positive women in 2005 confirmed by HPV genotyping were taken as an analytic cohort (AC), as shown in Fig. 1.

The differences of age were compared among women with low, medium, or high viral loads using one-way ANOVA method, and other categorical variables using Pearson Chi-square method. We analyzed the prevalence of cytological lesions in relation to type-specific HPV viral load. Cumulative incidence rate (CIR) of CIN2 + over ten-year follow-up by type-specific hrHPV viral loads was estimated using Kaplan-Meier methods. Hazard ratios were estimated using Cox proportional hazard models. The CIR of CIN2 + stratified by various viral load cutoffs, cytology grades, and VIA results among HPV16/18 positive women and non-16/18 hrHPV positive women were compared using Log-rank test. All statistical tests were twotailed with 0.05 as significance level and all analyses were performed using SAS 9.2.

\section{Results}

\subsection{Demographic characteristics}

Among 209 hrHPV-positive women at AC baseline, the average age was 45 years and sexual debut age was 21 years. The majority of 


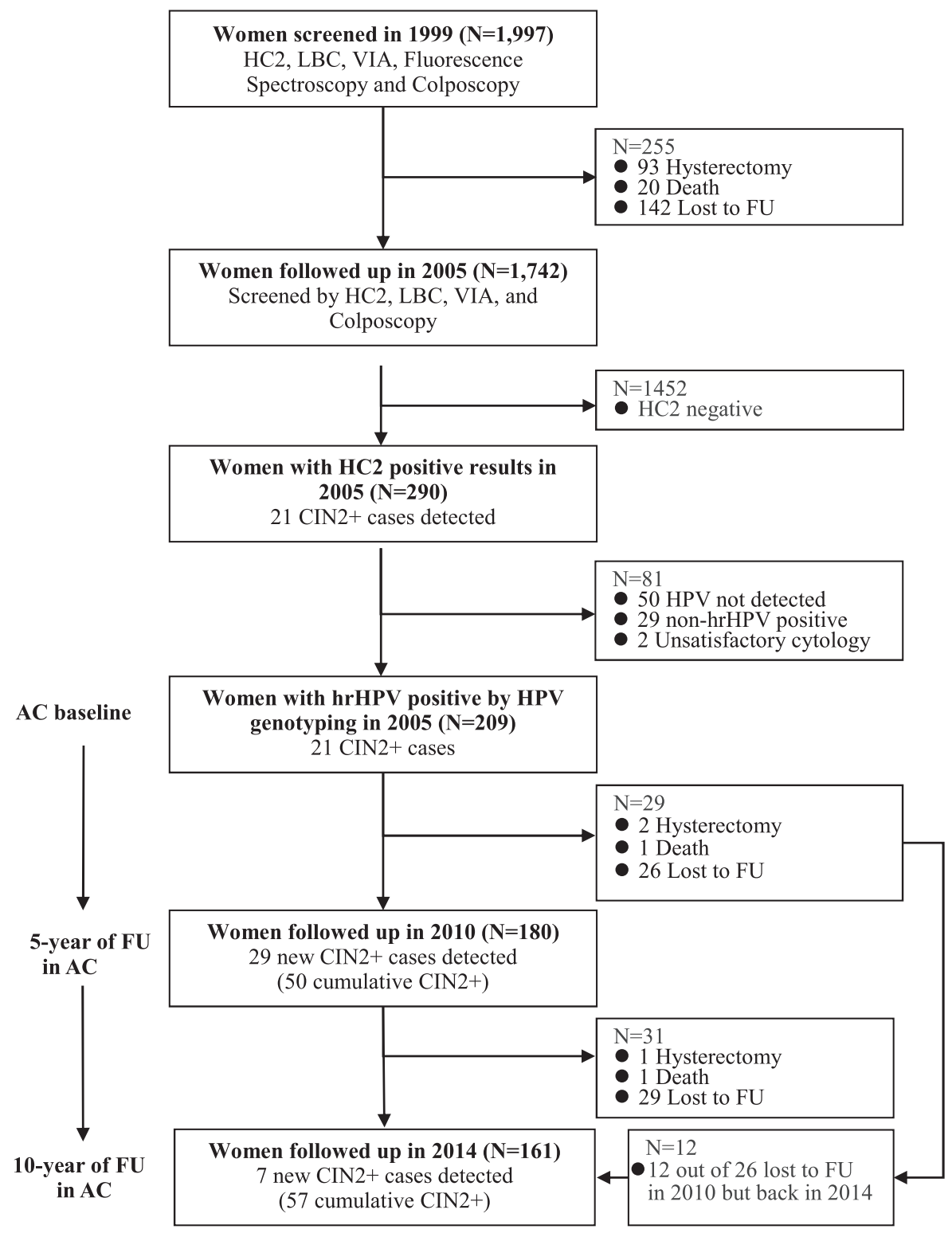

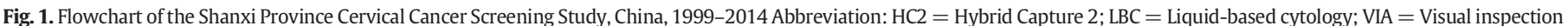
with acetic acid; HPV = Human papillomavirus; CIN2 + = Cervical intraepithelial neoplasia grade 2 or worse; AC = Analytic cohort; FU = Follow-up.

women received junior high school education or higher level, were nonsmokers, and premenopausal. Of these women, $4.3 \%$ reported their husband's extramarital sex and $13.7 \%$ reported their extramarital sex. One-third women washed vulvae before or after intercourse. Onefourth had four or more pregnancies, and one-half gave three fullterm births or more. Except for the number of marriages, no significant differences for other characteristics among women with different baseline AC viral load were found (Supplementary Table 1).

\subsection{Cervical cytological lesions in relation to baseline genotypes and viral load}

The baseline prevalence of atypical squamous cell of undetermined significance or higher (ASCUS +) among 209 hrHPV-positive women was positively correlated with baseline hrHPV viral load stratified by genotypes ( $p_{\text {trend }}<0.001$ ), as shown in Table 1 . The prevalence of ASCUS + increased with the incremental viral loads ranging from $41.7 \%$ to $82.9 \%$ among HPV16/18 positive women and from $37.2 \%$ to $68.6 \%$ among non16/18 hrHPV positive women. HPV 16 positive women with high viral loads had a significantly higher proportion of high squamous intraepithelial lesions (HSIL) (55.2\%) than those with low $(25.0 \%)$ or moderate viral loads $(38.7 \%)(p<0.05)$.

\subsection{Detection of CIN2 + cases by baseline genotypes and viral load}

The number of detected CIN2 + cases depended on both HPV genotypes and viral load, as shown in Table 2. The majority (96.5\%) of CIN2 + cases were infected with at least one type of HPV 16 (63.2\%), 58 (12.3\%), $31(12.3 \%), 18(3.5 \%), 33(7.0 \%), 45(3.5 \%)$, or 52 (5.3\%). Baseline CIN2+ cases were almost equally distributed among women with moderate viral load (42.9\%) and high viral load (57.1\%), by contrast, half of follow-up cases were mainly distributed among women with high viral load.

\subsection{CIR of CIN2 + by HPV genotypes and baseline viral load}

High viral load of specific hrHPV type in different viral species at baseline predicted an incremental ten-year CIR of CIN2 +, as illustrated 
Table 1

Distribution of abnormal cytology at analytic baseline stratified by type-specific HPV viral load in 209 hrHPV-positive women.

\begin{tabular}{|c|c|c|c|c|c|c|c|c|}
\hline \multirow[t]{2}{*}{ Alpha } & \multirow[t]{2}{*}{ HPV viral load at analytic baseline ${ }^{\mathrm{b}}$} & \multirow[t]{2}{*}{$\mathrm{N}$} & \multicolumn{5}{|c|}{ Cytology results at analytic baseline } & \multirow[t]{2}{*}{$p_{\text {trend }}{ }^{\mathrm{d}}$} \\
\hline & & & NILM \% & ASCUS \% & LSIL \% & HSIL \% & $\overline{\text { ASCUS }+\%}$ & \\
\hline \multirow[t]{3}{*}{ Any hrHPV } & Low & 67 & 61.2 & 17.9 & 9 & 11.9 & 38.8 & $<0.001$ \\
\hline & Moderate & 72 & 37.5 & 22.2 & 9.7 & 30.6 & 62.5 & \\
\hline & High & 70 & 24.3 & 17.1 & 24.3 & 34.3 & 75.7 & \\
\hline \multirow[t]{3}{*}{ HPV 16/18 } & Low & 24 & 58.3 & 20.8 & 0 & 20.8 & 41.7 & 0.001 \\
\hline & Moderate & 36 & 25 & 25 & 11.1 & 38.9 & 75.0 & \\
\hline & High & 35 & 17.1 & 17.1 & 17.1 & 48.6 & 82.9 & \\
\hline \multirow[t]{3}{*}{ Non-16/18 hrHPV } & Low & 43 & 62.8 & 16.3 & 14.0 & 7.0 & 37.2 & 0.006 \\
\hline & Moderate & 36 & 50.0 & 19.4 & 8.3 & 22.2 & 50.0 & \\
\hline & High & 35 & 31.4 & 17.1 & 31.4 & 20.1 & 68.6 & \\
\hline \multirow[t]{3}{*}{ HPV16/18/31/33/45/52/58 } & Low & 55 & 54.5 & 21.8 & 9.1 & 14.5 & 45.5 & 0.000 \\
\hline & Moderate & 64 & 34.4 & 21.9 & 9.4 & 34.4 & 65.6 & \\
\hline & High & 59 & 18.6 & 16.9 & 25.4 & 39.0 & 81.4 & \\
\hline \multirow[t]{3}{*}{ HPV 16} & Low & 20 & 50.0 & 25.0 & 0 & 25.0 & 50.0 & 0.002 \\
\hline & Moderate & 31 & 29 & 19.4 & 12.9 & 38.7 & 71.0 & \\
\hline & High & 29 & 10.3 & 13.8 & 20.7 & 55.2 & 89.7 & \\
\hline \multirow[t]{3}{*}{ HPV $18^{\mathrm{a}}$} & Low & 4 & 100 & 0 & 0 & 0 & 0 & 0.230 \\
\hline & Moderate & 5 & 0 & 60 & 0 & 40 & 100 & \\
\hline & High & 6 & 50.0 & 33.3 & 0 & 16.7 & 50.0 & \\
\hline \multirow[t]{3}{*}{ HPV $31^{a}$} & Low & 5 & 60.0 & 20.0 & 20.0 & 0 & 40.0 & 0.500 \\
\hline & Moderate & 7 & 28.6 & 28.6 & 0 & 42.9 & 71.4 & \\
\hline & High & 8 & 37.5 & 12.5 & 25.0 & 25.0 & 62.5 & \\
\hline \multirow[t]{3}{*}{ HPV $33^{\mathrm{a}}$} & Low & 9 & 55.6 & 11.1 & 33.3 & 0 & 44.4 & 0.075 \\
\hline & Moderate & 7 & 42.9 & 28.6 & 0 & 28.6 & 57.1 & \\
\hline & High & 8 & 12.5 & 37.5 & 37.5 & 12.5 & 87.5 & \\
\hline \multirow[t]{3}{*}{ HPV $52^{\mathrm{a}}$} & Low & 14 & 42.9 & 35.7 & 7.1 & 14.3 & 57.1 & 0.552 \\
\hline & Moderate & 13 & 61.5 & 15.4 & 7.7 & 15.4 & 38.5 & \\
\hline & High & 4 & 50 & 0 & 25.0 & 25.0 & 50.0 & \\
\hline \multirow[t]{3}{*}{ HPV $58^{a}$} & Low & 7 & 57.1 & 14.3 & 14.3 & 14.3 & 42.9 & 0.082 \\
\hline & Moderate & 7 & 14.3 & 28.6 & 14.3 & 42.9 & 85.7 & \\
\hline & High & 11 & 18.2 & 0 & 45.5 & 36.4 & 81.9 & \\
\hline
\end{tabular}

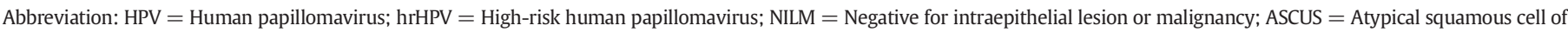

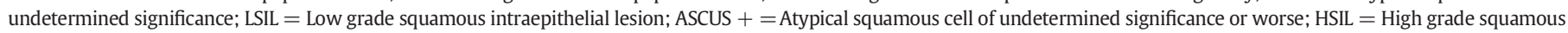
intraepithelial lesion;

a Women concurrently infected with HPV 16 were not included in the final analysis.

b HPV viral load was categorized by RLU/CO ratio: 1-9.99 RLU/CO as low; 10-99.99 RLU/CO as moderate; $\geq 100$ RLU/CO as high.

c HSIL included four cases of ASCUS-H.

d $p_{\text {trend }}$ was calculated using Chi-square methods to compare the proportions of ASCUS + with increased viral loads from low to high level stratified by hrHPV types.

by Fig. 2. Among any alpha-9 species positive women, a striking risk stratification of CIN2 + with HR of 4.6 (95\% CI: 2.0 to 10.6) was seen between women with low and high viral load with CIR of $16.2 \%$ and $59.2 \%$, respectively. By contrast, no notable stratification was observed for any alpha-7 species positive women. Significant risk stratification was also observed when all non-16/18 types were combined ( $\mathrm{HR}=$ $7.3,95 \% \mathrm{CI}=1.6$ to 32.8 ) with $\mathrm{CIR}$ of $5.1 \%$ versus $35.5 \%$. The trend of risk stratifications by type-specific hrHPV viral loads was similar even after CIN2 + cases at baseline were excluded from 209 hrHPV-positive women to estimate incident CIN2 + (Supplementary Fig. 1).

\subsection{CIR of CIN2 + by triage methods}

The CIRs of CIN2 + among HPV 16/18 and non-16/18 hrHPV positive women were statistically stratified by ASCUS + and viral load cutoffs (Fig. 3). Among HPV 16/18-positive women, ten-year CIR of CIN2 + was high albeit with normal cytology (15.3\%), normal VIA (32.4\%), viral load with RLU/CO $<10$ (23.6\%) or $\mathrm{RLU} / \mathrm{CO}<100$ (33.8\%). Among non-16/18 hrHPV positive women, ten-year CIR of CIN2 + was significantly stratified by cytology grade of ASCUS + (2.0\% VS. $34.6 \%)$, viral load cutoffs at $10 \mathrm{RLU} / \mathrm{CO}$

Table 2

Cumulative detection of CIN2 + in 209 hrHPV-positive women, stratified by timing of detection, by HPV viral load.

\begin{tabular}{|c|c|c|c|c|c|c|c|c|c|c|c|c|}
\hline \multirow[t]{3}{*}{ HPV infection status at AC baseline } & \multicolumn{4}{|c|}{ AC baseline CIN2 + cases $(N=21) \%$} & \multicolumn{4}{|c|}{$\begin{array}{l}\text { AC new cases of CIN } 2+\text { over } 10 \text {-year of } \\
\text { FU }(N=36) \%\end{array}$} & \multicolumn{4}{|c|}{$\begin{array}{l}\text { AC accumulative cases of CIN2 }+ \text { at } \\
\text { baseline and } 10 \text {-year of FU }(N=57) \%\end{array}$} \\
\hline & \multirow[t]{2}{*}{ Overall } & \multicolumn{3}{|c|}{ HPV viral loads ${ }^{\mathrm{b}}$} & \multirow[t]{2}{*}{ Overall } & \multicolumn{3}{|c|}{ HPV viral loads ${ }^{\mathrm{b}}$} & \multirow[t]{2}{*}{ Overall } & \multicolumn{3}{|c|}{ HPV viral loads ${ }^{\mathrm{b}}$} \\
\hline & & Low & Moderate & High & & Low & Moderate & High & & Low & Moderate & High \\
\hline Any hrHPV & 100 & 0 & 42.9 & 57.1 & 100 & 19.5 & 30.6 & 50.0 & 100 & 12.3 & 35.1 & 52.6 \\
\hline HPV 16/18 & 71.4 & 0 & 28.6 & 42.8 & 63.9 & 13.9 & 22.2 & 27.8 & 66.7 & 8.8 & 24.6 & 33.3 \\
\hline Non-16/18 hrHPV & 28.6 & 0 & 14.3 & 14.3 & 36.1 & 5.6 & 8.3 & 22.2 & 33.3 & 3.5 & 10.5 & 19.3 \\
\hline HPV16/18/31/33/45/52/58 & 95.2 & 0 & 42.9 & 52.4 & 97.2 & 19.4 & 30.6 & 47.2 & 96.5 & 12.3 & 35.1 & 49.1 \\
\hline HPV 16 & 71.4 & 0 & 28.6 & 42.8 & 58.3 & 13.9 & 16.7 & 27.8 & 63.2 & 8.8 & 21.1 & 33.3 \\
\hline HPV $18^{a}$ & 0 & 0 & 0 & 0 & 5.6 & 0 & 5.6 & 0 & 3.5 & 0 & 3.5 & 0 \\
\hline HPV $31^{a}$ & 9.8 & 0 & 4.9 & 4.9 & 13.9 & 0 & 5.6 & 8.3 & 12.3 & 0 & 5.3 & 7.0 \\
\hline HPV $33^{a}$ & 4.8 & 0 & 0 & 4.8 & 8.3 & 2.8 & 0 & 5.6 & 7.0 & 1.8 & 0 & 5.3 \\
\hline HPV $52^{\mathrm{a}}$ & 0 & 0 & 0 & 0 & 8.3 & 0 & 5.6 & 2.8 & 5.3 & 0 & 3.5 & 1.8 \\
\hline HPV $58^{a}$ & 19.0 & 0 & 9.5 & 9.5 & 8.3 & 2.8 & 0 & 5.6 & 12.3 & 1.8 & 3.5 & 7.0 \\
\hline
\end{tabular}

Abbreviation: HPV = Human papillomavirus; hrHPV = High-risk HPV; CIN2 + = Cervical intraepithelial neoplasia 2 or worse; AC = Analytic cohort; FU = Follow-up.

a Women concurrently infected with HPV 16 were not included in the final analysis.

b HPV viral load was categorized by RLU/CO ratio: 1-9.99 RLU/CO as low; 10-99.99 RLU/CO as moderate; $\geq 100$ RLU/CO as high. 
(5.1\% VS. 27.2\%), at $100 \mathrm{RLU} / \mathrm{CO}$ (11.0\% VS. 35.5\%), but not by VIA (19.1\% VS. 19.0\%).

\section{Discussion}

Our study is among the first to assess the optimum triage strategies of HPV 16/18 positive women and non-16/18 hrHPV positive by typespecific HPV viral loads, in comparison to cytology grade and VIA in a population-based cervical cancer screening cohort. We found that HPV16/18 positive women had a high risk of CIN2 + even with a low viral load or normal cytology and non-16/18 hrHPV positive women has a striking risk stratification by cytology and suitable viral load cutoffs. However, VIA may not be a viable risk triage tool for non-16/18 hrHPV positive women.

We evaluated the association between hrHPV viral load and prevalent cytological and histological lesions, and the predictive value of CIN2 + by hrHPV genotypes. Among 13 hrHPV genotypes, HPV 16 viral load had the strongest dose-dependent effect with cytological lesions and $\mathrm{CIN} 2+$, which supported existing evidences on the varied carcinogenicity of type-specific hrHPV [6,27], and was also in line with other studies that HPV 16 viral load can stratify the prevalent risks of cytological lesions and the risk of CIN and cancers [17,28-30]. Moreover, consistent with the US ALTS trial [31], we also found that the elevated risks of CIN2 + for high viral loads appeared to be specific to alpha-9 associated types, although our study power was rather limited for more rare HPV type assessments.
In regard to HPV 18, the association of viral load with the risk of high-grade CIN was not consistent among studies [32-34]. Our current data showed that all of incident CIN $2+$ cases among HPV 18 positive women were infected with moderate viral load, rather than high load, albeit our limited sample size to evaluate this association. This finding indicated that HPV 18-induced cervical lesions was more likely to be associated with increased HPV transcript rather than viral copies, [35] more integrated than episome status in the invasive cancer [36]. Besides, HPV 18, as a representative type of alpha-7 species, was found to be strongly associated with adenocarcinoma [37-39]. The glandular epithelium of adenocarcinoma does not support productive HPV infection, therefore, the low viral load in this type of malignancy results in the difficulty of HPV detection and the underestimation of the association of HPV 18 loads with glandular lesions [40].

Among non-16/18 hrHPV genotypes, special attention should be given to HPV 31 and HPV 33 due to their high carcinogenicity $[6,41]$, and to HPV 52 and 58 due to their high prevalence in Asian women [42]. We noticed that these four genotypes had a substantially higher risk of CIN2 + over the follow-up than at baseline, indicating that a long time was needed to progress from infection to high-grade CIN compared with HPV16, which was consistent with other studies [19]. Although the trends of prevalent cytological lesions in positive relation to viral load of these genotypes could be seen, the increasing risk of CIN2 + was unable to reach statistical significance. The observed trends might not be due to random variation, because prevalent cytology lesions or prospective risk of CINs in women with the increased high

\begin{tabular}{|c|c|c|}
\hline Species & HPV types & $\begin{array}{c}\text { AC baseline } \\
\text { HPV viral load } \dagger\end{array}$ \\
\hline 9 & HPV 16 & $\begin{array}{l}\text { Low } \\
\text { Moderate } \\
\text { High }\end{array}$ \\
\hline 9 & HPV $31^{*}$ & $\begin{array}{l}\text { Low } \\
\text { Moderate } \\
\text { High }\end{array}$ \\
\hline 9 & HPV $33^{*}$ & $\begin{array}{l}\text { Low } \\
\text { Moderate } \\
\text { High }\end{array}$ \\
\hline 9 & HPV $52^{*}$ & $\begin{array}{l}\text { Low } \\
\text { Moderate } \\
\text { High }\end{array}$ \\
\hline 9 & HPV $58^{*}$ & $\begin{array}{l}\text { Low } \\
\text { Moderate } \\
\text { High }\end{array}$ \\
\hline 9 & all & $\begin{array}{l}\text { Low } \\
\text { Moderate } \\
\text { High }\end{array}$ \\
\hline 9 & non-HPV 16 & $\begin{array}{l}\text { Low } \\
\text { Moderate } \\
\text { High }\end{array}$ \\
\hline 7 & HPV $18^{*}$ & $\begin{array}{l}\text { Low } \\
\text { Moderate } \\
\text { High }\end{array}$ \\
\hline 7 & all & $\begin{array}{l}\text { Low } \\
\text { Moderate } \\
\text { High }\end{array}$ \\
\hline 7 & non-HPV 18 & $\begin{array}{l}\text { Low } \\
\text { Moderate } \\
\text { High }\end{array}$ \\
\hline
\end{tabular}

\author{
CIR of CIN2+ \\ $(\mathbf{9 5} \% \mathrm{CI})$ \\ 27.6(9.9,48.8) \\ $40.6(23.0,57.5)$ \\ $70.4(48.6,84.4)$ \\ 0 \\ $42.9(9.8,73.4)$ \\ $63.5(17.1,89.0)$ \\ $16.7(0.8,51.7)$ \\ 0 \\ $40.0(5.2,75.3)$ \\ 0 \\ $16.7(2.7,41.3)$ \\ $50.0(0.6,91.0)$ \\ $14.3(0.7,46.5)$ \\ 28.6(4.1,61.2) \\ $38.6(11.7,65.6)$ \\ $16.2(7.1,28.7)$ \\ $33.3(21.1,46.0)$ \\ $59.2(43.4,72.0)$ \\ 7.7 $(1.3,21.7)$ \\ $24.1(9.7,42.1)$ \\ $44.9(22.6,64.9)$ \\ $\stackrel{0}{40.0(5.2,75.3)}$ \\ 0 \\ 0 \\ $25.0(3.7,55.8)$ \\ $16.9(2.6,42.0)$ \\ 0 \\ 0 \\ 28.6(4.1,61.2)
}

HR( $95 \%$

1 (reference)

$1.8(0.6,5.2)$

$3.7(1.4,9.8)$

1(reference)

$1.1(0.2,4.8)$

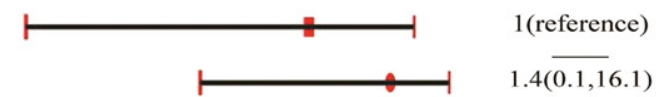

0.657

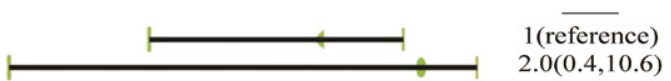

0.192

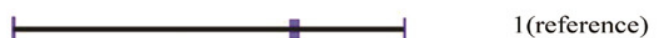

$2.9(0.3,32.3)$

3.0 $(0.3,26.7)$

1(reference)

$2.5(1.1,6.0)$

$4.6(2.0,10.6)$

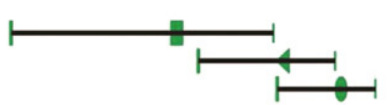

1(reference)

3.7 $(0.8,18.5)$

$5.9(1.3,27.4)$

0.030

0.214

1(reference)

0.328

1 (reference)

$0.7(0.1,5.3)$

0.249

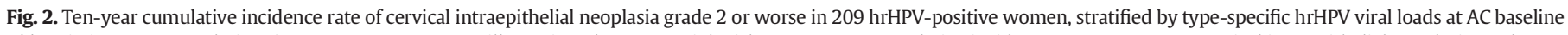

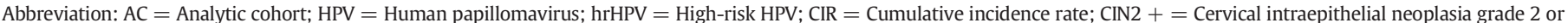

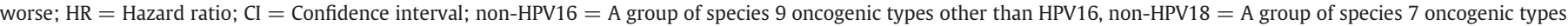

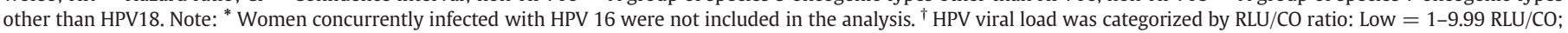
Moderate $=10-99.99 \mathrm{RLU} / \mathrm{CO}$; High $=100$ or higher RLU/CO; $\ddagger$ Moderate viral load was taken as the reference to evaluate the HR of high viral load. The X-axis is logarithmic. 


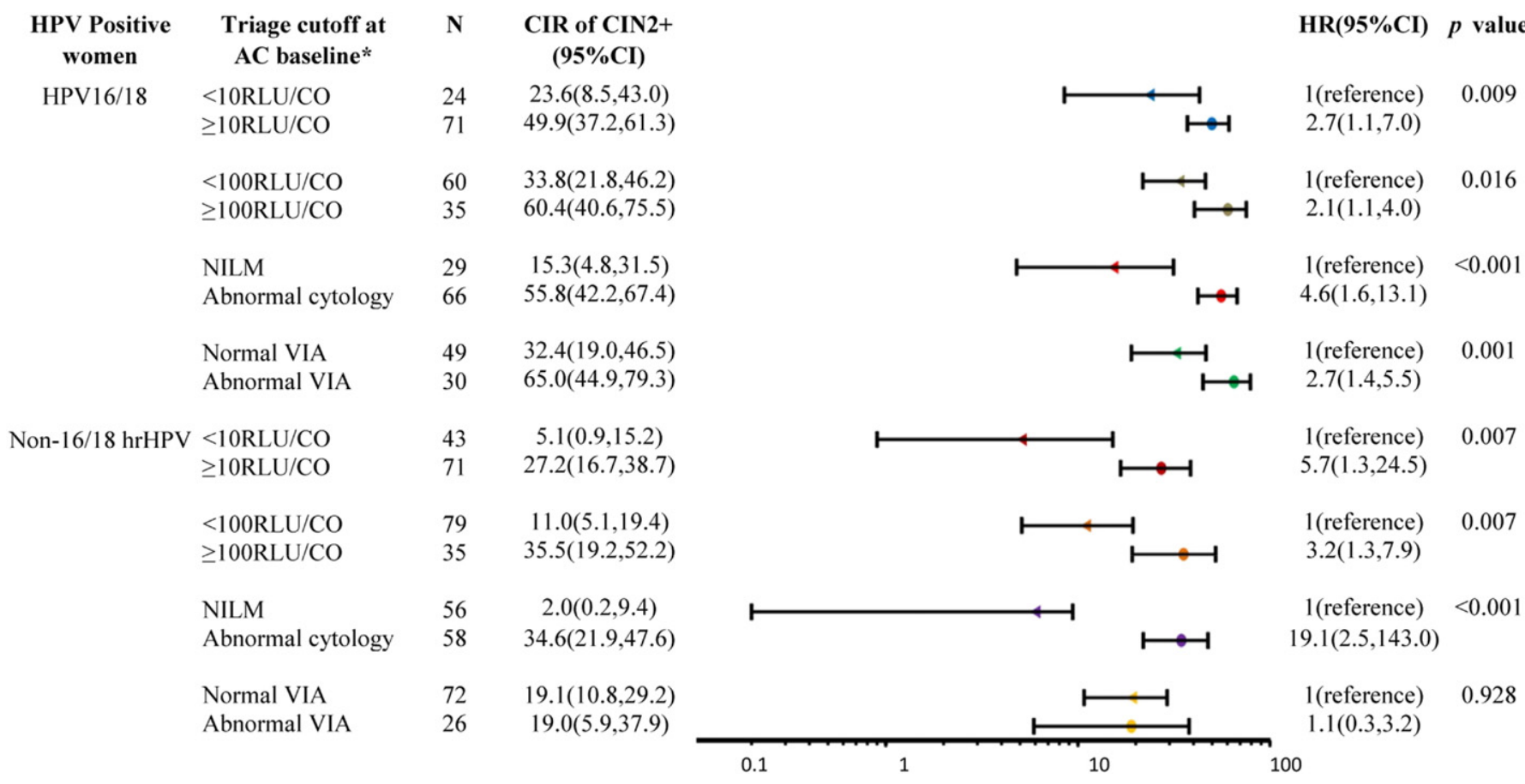

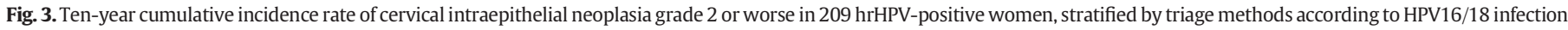

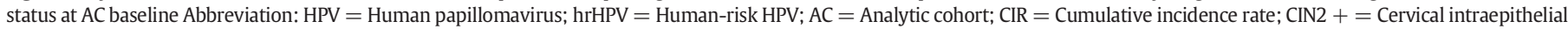

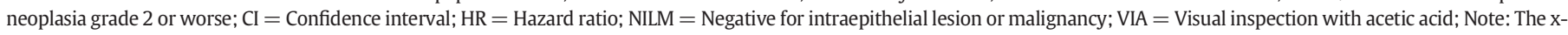
axis is logarithmic.

non-16/18 hrHPV viral loads have been reported by previous studies $[31,43]$. However, considering different sensitivities among methods of measuring viral loads [38], further studies involving larger sample sizes of HPV 31, 33, 52 and 58 positive women and measurement of viral load by sensitive quantitative methods, such as RT-PCR, are warranted.

HPV life cycle strictly follows the differentiation program of cellular DNA machinery. The viral load reflects the increase of the number of infected cells or viral copies in individual cells. Therefore, the possible mechanism of high viral load associated with the incremental risk of cervical neoplasia was an elevated likelihood of having cells with transforming infections and viral integrating into cellular genome as increasing numbers of infected cells and/or increasing numbers of viral copies in individual cells $[44,45]$. Moreover, the duration of persistent high viral load rather than baseline viral load determined the extent of this likelihood, as supposed by our previous study and and Gravitt Patti E.'s study [17,21].

HPV 16/18 positive women were recommended for direct referral to colposcopy and non-16/18 hrHPV positive women were recommended for triaging with cytology to colposcopy according to the updated guidelines [9]. Our data showed that HPV16/18 positive women, even with normal cytology, normal VIA, or low viral load still suffered from a notably high risk of CIN2 +. This finding supports the ASCCP guidelines in managing the HPV16/18 positive women to avoid missed diagnosis of CIN2 + cases that might occur with further triage [9]. On the other hand, among non-16/18 hrHPV positive women, the CIR of CIN2+ could be stratified by a cytology grade of ASCUS + and by viral load cutoffs, but not by VIA. This data suggests that triaging non-16/18 hrHPVpositive women to colposcopy using a suitable viral load cutoff could be a feasible option in low-resource areas without cytology screening.

2013 WHO guidelines recommended HPV testing followed by VIA triage in underserved countries [46]. Therefore, the feasibility of triaging HPV16/18 positive women and non-16/18 hrHPV-positive women using VIA was evaluated in our study. Risk stratification by VIA was only significant among HPV16/18 positive women, but was not obvious among non-16/18 hrHPV positive women. Therefore, our data does not support using VIA as a triaging tool for non-16/18 hrHPV-positive women. This finding could be explained by a previous finding by Jeronimo J et that VIA was less sensitive for non-16/18 HPV than for HPV 16/18, since HPV 16 was most likely to cause robust visual (aceto-white) changes [47].

This present findings of HPV viral loads as an alternative to cytology in stratifying the risk of high-grade CIN contributed valuable data for future discussions related to the potential application of viral load measurement to increase test specificity in cervical cancer screening. The well-organized follow-up of the population by both active follow-up through three organized screenings and passive follow-up through a nationwide cancer registry in our study ensured minimal missing reports of cervical cancer and precursors cases over ten years. Nevertheless, several limitations are also needed to address. Firstly, the age range of participants at baseline was narrow, which covers the natural age range of peri-menopause for Chinese women [48]. Therefore, cautions should be taken in applying this study's conclusions to younger women. Secondly, small sample size of individual rare types-positive women stratified by viral loads limited our statistical power to draw definitive conclusions regarding disease progression. However, the trends point to the need for further investigation. Finally, viral load was estimated using RLU/CO in HC2 assay in a semi-quantitative method, therefore the absolute risk of cervical cytology and histology related with viral loads may not be directly comparable to other studies. Nevertheless, HC2 RLU/CO viral load measurement was found to correlate well with real-time PCR viral load measurement [25]. Meanwhile, semiquantitative crude estimation does not affect the generalizability about the validity of viral loads in comparison to cytology and VIA that were derived from the same risk estimation standard.

Because of comparable clinical performance, independence from provider's experience and lower economic cost compared with cytology, HPV viral load testing has the potential application as an alternative of cytology in future clinical practice, especially in the regions with cytology inaccessible, although the extent to which these findings can be transformed into clinical practice must be rigorously verified in the context of viral load measurement methods and the variation of 
type-specific carcinogenicity among regions. Furthermore, HPV viral load as a triage method, if measured by an assay which could be read simultaneously with HPV test, would potentially minimize the financial dependence for additional sampling and biomarker testing as required by cytology.

In conclusion, our study showed a potential role of viral load in stratifying the risk for hrHPV positive women, provided consistent evidences with ASCCP guidelines of directly referring HPV 16/18 positive women to colposcopy, and suggested triaging non-16/18 hrHPV positive women using viral loads in resource-limited countries. This present finding from population-baesd cohort data will benefit some countries with comparable settings with China. Nevertheless, more large scale population trials from different settings or regions are needed to ensure the clinical performance before HPV DNA viral load as a triage method was transformed into clinical practice globally.

Supplementary data to this article can be found online at https://doi. org/10.1016/j.ygyno.2017.11.016.

\section{Conflict of interest statement}

All authors have no conflict of interest except Dr. Smith reports grants and non-financial support from Trovagene, grants and personal fees from Hologic, grants and personal fees from BD, non-financial support from Arbor Vita, outside the submitted work.

\section{Acknowledgements}

The authors wish to thank keith Allan Clark II for his careful editing of this manuscript. This work was supported by the National Natural Science Foundation of China (Grant number: 81322040, 81050018) and the CAMS Initiative for Innovative Medicine (2016-I2M-1-019).

\section{Authors' contributions} Wang.

Conception and design: Y.L. Qiao, F.H. Zhao, J.S. Smith, L. Dong, M.

Lab work: L. Dong, Q. Zhang, X.L. Zhao.

Acquisition of clinical data: X.L. Zhao, S.Y. Hu, F.H. Zhao.

Analysis and interpretation of data: L. Dong, M. Wang, X.L. Zhao, J.S. Smith, R.M. Feng, F.H. Zhao, Y.L. Qiao.

Writing, review and/or revision of the manuscript: M. Wang, L. Dong, X.L. Zhao, R.M. Feng, Q. Zhang, S.Y. Hu, J.S. Smith, F.H. Zhao, Y.L. Qiao.

Study supervision: F.H. Zhao, Y.L. Qiao.

\section{References}

[1] J.M. Walboomers, M.V. Jacobs, M.M. Manos, F.X. Bosch, J.A. Kummer, K.V. Shah, et al., Human papillomavirus is a necessary cause of invasive cervical cancer worldwide, J. Pathol. 189 (1999) 12-19.

[2] J.E. Tota, J. Bentley, J. Blake, F. Coutlee, M.A. Duggan, A. Ferenczy, et al., Introduction of molecular HPV testing as the primary technology in cervical cancer screening: acting on evidence to change the current paradigm, Prev. Med. 98 (2017) 5-14.

[3] J.B. Lew, K. Simms, M. Smith, H. Lewis, H. Neal, K. Canfell, Effectiveness modelling and economic evaluation of primary HPV screening for cervical cancer prevention in New Zealand, PLoS One 11 (2016), e0151619.

[4] G.Y. Ho, R. Bierman, L. Beardsley, C.J. Chang, R.D. Burk, Natural history of cervicovaginal papillomavirus infection in young women, N. Engl. J. Med. 338 (1998) 423-428

[5] M. Arbyn, S. de Sanjose, M. Saraiya, M. Sideri, J. Palefsky, C. Lacey, et al., EUROGIN 2011 roadmap on prevention and treatment of HPV-related disease, Int. J. Cancer 131 (2012) 1969-1982.

[6] M. Schiffman, A.G. Glass, N. Wentzensen, B.B. Rush, P.E. Castle, D.R. Scott, et al., A long-term prospective study of type-specific human papillomavirus infection and risk of cervical neoplasia among 20,000 women in the Portland Kaiser Cohort Study, Cancer Epidemiol. Biomark. Prev. 20 (2011) 1398-1409.

[7] T.C. Wright, M.H. Stoler, C.M. Behrens, A. Sharma, G. Zhang, T.L. Wright, Primary cervical cancer screening with human papillomavirus: end of study results from the ATHENA study using HPV as the first-line screening test, Gynecol. Oncol. 136 (2015) 189-197.

[8] A. Clad, M. Reuschenbach, J. Weinschenk, R. Grote, J. Rahmsdorf, N. Freudenberg, Performance of the Aptima high-risk human papillomavirus mRNA assay in a referral population in comparison with Hybrid Capture 2 and cytology, J. Clin. Microbiol. 49 (2011) 1071-1076.
9] W.K. Huh, K.A. Ault, D. Chelmow, D.D. Davey, R.A. Goulart, F.A. Garcia, et al., Use of primary high-risk human papillomavirus testing for cervical cancer screening: interim clinical guidance, Gynecol. Oncol. 136 (2015) 178-182.

[10] F.H. Zhao, M.J. Lin, F. Chen, Hu SY, R. Zhang, J.L. Belinson, et al., Performance of highrisk human papillomavirus DNA testing as a primary screen for cervical cancer: a pooled analysis of individual patient data from 17 population-based studies from China, Lancet Oncol. 11 (2010) 1160-1171.

[11] C. Sauvaget, J.M. Fayette, R. Muwonge, R. Wesley, R. Sankaranarayanan, Accuracy of visual inspection with acetic acid for cervical cancer screening, Int. J. Gynaecol. Obstet. 113 (2011) 14-24.

[12] A.T. Lorincz, P.E. Castle, M.E. Sherman, D.R. Scott, A.G. Glass, S. Wacholder, et al., Viral load of human papillomavirus and risk of CIN3 or cervical cancer, Lancet 360 (2002) 228-229.

[13] M.C. Abba, S.A. Mouron, M.A. Gomez, F.N. Dulout, C.D. Golijow, Association of human papillomavirus viral load with HPV16 and high-grade intraepithelial lesion, Int. J. Gynecol. Cancer 13 (2003) 154-158.

[14] M. Schiffman, R. Herrero, A. Hildesheim, M.E. Sherman, M. Bratti, S. Wacholder, et al., HPV DNA testing in cervical cancer screening: results from women in a high-risk province of Costa Rica, JAMA 283 (2000) 87-93.

[15] C.A. Sun, H.C. Lai, C.C. Chang, S. Neih, Yu CP, T.Y. Chu, The significance of human papillomavirus viral load in prediction of histologic severity and size of squamous intraepithelial lesions of uterine cervix, Gynecol. Oncol. 83 (2001) 95-99.

[16] H.T. Tsai, Wu CH, H.L. Lai, R.N. Li, Y.C. Tung, H.Y. Chuang, et al., Association between quantitative high-risk human papillomavirus DNA load and cervical intraepithelial neoplasm risk, Cancer Epidemiol. Biomark. Prev. 14 (2005) 2544-2549.

[17] P.E. Gravitt, M.B. Kovacic, R. Herrero, M. Schiffman, C. Bratti, A. Hildesheim, et al., High load for most high risk human papillomavirus genotypes is associated with prevalent cervical cancer precursors but only HPV16 load predicts the development of incident disease, Int. J. Cancer 121 (2007) 2787-2793.

[18] L. Del Rio-Ospina, S.C. Soto-De Leon, M. Camargo, D.A. Moreno-Perez, R. Sanchez, A. Perez-Prados, et al., The DNA load of six high-risk human papillomavirus types and its association with cervical lesions, BMC Cancer 15 (2015) 100.

[19] C.E. Depuydt, A.M. Criel, I.H. Benoy, M. Arbyn, A.J. Vereecken, J.J. Bogers, Changes in type-specific human papillomavirus load predict progression to cervical cancer, J. Cell. Mol. Med. 16 (2012) 3096-3104.

[20] C.E. Depuydt, J. Jonckheere, M. Berth, G.M. Salembier, A.J. Vereecken, J.J. Bogers, Serial type-specific human papillomavirus (HPV) load measurement allows differentiation between regressing cervical lesions and serial virion productive transient infections, Cancer Med. 4 (2015) 1294-1302.

[21] S.M. Wang, D. Colombara, J.F. Shi, F.H. Zhao, J. Li, F. Chen, et al., Six-year regression and progression of cervical lesions of different human papillomavirus viral loads in varied histological diagnoses, Int. J. Gynecol. Cancer 23 (2013) 716-723.

[22] J. Belinson, Y.L. Oiao, R. Pretorius, W.H. Zhang. P. Elson, L. Li, et al., Shanxi Province Cervical Cancer Screening Study: a cross-sectional comparative trial of multiple techniques to detect cervical neoplasia, Gynecol. Oncol. 83 (2001) 439-444.

[23] J.F. Shi, J.L. Belinson, F.H. Zhao, R.G. Pretorius, J. Li, J.F. Ma, et al., Human papillomavirus testing for cervical cancer screening: results from a 6-year prospective study in rural China, Am. J. Epidemiol. 170 (2009) 708-716.

[24] Q. Zhang, L. Dong, S. Hu, R. Feng, X. Zhang, Q. Pan, et al., Risk stratification and longterm risk prediction of E6 oncoprotein in a prospective screening cohort in China, Int. J. Cancer 141 (2017) 1110-1119.

[25] P.E. Gravitt, R.D. Burk, A. Lorincz, R. Herrero, A. Hildesheim, M.E. Sherman, et al., A comparison between real-time polymerase chain reaction and hybrid capture 2 for human papillomavirus DNA quantitation, Cancer Epidemiol. Biomark. Prev. 12 (2003) 477-484

[26] V. Dalstein, D. Riethmuller, J.L. Pretet, K. Le Bail Carval, J.L. Sautiere, J.P. Carbillet, et al., Persistence and load of high-risk HPV are predictors for development of high-grade cervical lesions: a longitudinal French cohort study, Int. J. Cancer 106 (2003) 396-403.

[27] M.J. Khan, P.E. Castle, A.T. Lorincz, S. Wacholder, M. Sherman, D.R. Scott, et al., The elevated 10-year risk of cervical precancer and cancer in women with human papillomavirus (HPV) type 16 or 18 and the possible utility of type-specific HPV testing in clinical practice, J. Natl. Cancer Inst. 97 (2005) 1072-1079.

[28] P.E. Castle, A.C. Rodriguez, R.D. Burk, R. Herrero, S. Wacholder, M. Alfaro, et al., Short term persistence of human papillomavirus and risk of cervical precancer and cancer: population based cohort study, BMJ b2569 (2009) 339.

[29] A. Trevisan, N.F. Schlecht, A.V. Ramanakumar, L.L. Villa, E.L. Franco, Human papillomavirus type 16 viral load measurement as a predictor of infection clearance, J. Gen. Virol. 94 (2013) 1850-1857.

[30] J. Briolat, V. Dalstein, M. Saunier, K. Joseph, S. Caudroy, J.L. Pretet, et al., HPV prevalence, viral load and physical state of HPV-16 in cervical smears of patients with different grades of CIN, Int. J. Cancer 121 (2007) 2198-2204.

[31] L. Fu Xi, M. Schiffman, Y. Ke, J.P. Hughes, D.A. Galloway, Z. He, et al., Type-dependent association between risk of cervical intraepithelial neoplasia and viral load of oncogenic human papillomavirus types other than types 16 and 18, Int. J. Cancer 140 (2017) 1747-1756.

[32] L.T. Thomsen, K. Frederiksen, C. Munk, J. Junge, T. Iftner, S.K. Kjaer, Long-term risk of cervical intraepithelial neoplasia grade 3 or worse according to high-risk human papillomavirus genotype and semi-quantitative viral load among 33,288 women with normal cervical cytology, Int. J. Cancer 137 (2015) 193-203.

[33] A.T. Hesselink, J. Berkhof, D.A. Heideman, N.W. Bulkmans, J.E. van Tellingen, C.J. Meijer, et al., High-risk human papillomavirus DNA load in a population-based cervical screening cohort in relation to the detection of high-grade cervical intraepithelial neoplasia and cervical cancer, Int. J. Cancer 124 (2009) 381-386. 
[34] L.F. Xi, L.A. Koutsky, P.E. Castle, C.M. Wheeler, D.A. Galloway, C. Mao, et al., Human papillomavirus type 18 DNA load and 2-year cumulative diagnoses of cervical intraepithelial neoplasia grades 2-3, J. Natl. Cancer Inst. 101 (2009) 153-161.

[35] C.M. Ho, B.H. Lee, S.F. Chang, T.Y. Chien, S.H. Huang, C.C. Yan, et al., Type-specific human papillomavirus oncogene messenger RNA levels correlate with the severity of cervical neoplasia, Int. J. Cancer 127 (2010) 622-632.

[36] R.J. Kurman, M.H. Schiffman, W.D. Lancaster, R. Reid, A.B. Jenson, G.F. Temple, et al., Analysis of individual human papillomavirus types in cervical neoplasia: a possible role for type 18 in rapid progression, Am. J. Obstet. Gynecol. 159 (1988) 293-296.

[37] I. Hadzisejdc, M. Krasevic, H. Haller, B. Grahovac, Distribution of human papillomavirus types in different histological subtypes of cervical adenocarcinoma, Coll. Antropol. 31 (Suppl. 2) (2007) 97-102.

[38] M.A. Duggan, J.L. Benoit, S.E. McGregor, M. Inoue, J.G. Nation, G.C. Stuart, Adenocarcinoma in situ of the endocervix: human papillomavirus determination by dot blot hybridization and polymerase chain reaction amplification, Int. J. Gynecol. Pathol. 13 (1994) 143-149.

[39] G.M. Clifford, J.S. Smith, M. Plummer, N. Munoz, S. Franceschi, Human papillomavirus types in invasive cervical cancer worldwide: a meta-analysis, Br. J. Cancer 88 (2003) 63-73.

[40] G.D. Zielinski, P.J. Snijders, L. Rozendaal, N.F. Daalmeijer, E.K. Risse, F.J. Voorhorst, et al., The presence of high-risk HPV combined with specific p53 and p16INK4a expression patterns points to high-risk HPV as the main causative agent for adenocarcinoma in situ and adenocarcinoma of the cervix, J. Pathol. 201 (2003) 535-543.

[41] N. Munoz, F.X. Bosch, S. de Sanjose, R. Herrero, X. Castellsague, K.V. Shah, et al., Epidemiologic classification of human papillomavirus types associated with cervical cancer, N. Engl. J. Med. 348 (2003) 518-527.
[42] R. Wang, X.L. Guo, G.B. Wisman, E. Schuuring, W.F. Wang, Z.Y. Zeng, et al., Nationwide prevalence of human papillomavirus infection and viral genotype distribution in 37 cities in China, BMC Infect. Dis. 15 (2015) 257-266.

[43] M.B. Kovacic, P.E. Castle, R. Herrero, M. Schiffman, M.E. Sherman, S. Wacholder, et al. Relationships of human papillomavirus type, qualitative viral load, and age with cytologic abnormality, Cancer Res. 66 (2006) 10112-10119.

[44] P. Peitsaro, B. Johansson, S. Syrjanen, Integrated human papillomavirus type 16 is frequently found in cervical cancer precursors as demonstrated by a novel quantitative real-time PCR technique, J. Clin. Microbiol. 40 (2002) 886-891.

[45] J.M.L. Brotherton, S.N. Tabrizi, S. Phillips, J. Pyman, A.M. Cornall, N. Lambie, et al., Looking beyond human papillomavirus (HPV) genotype 16 and 18: defining HPV genotype distribution in cervical cancers in Australia prior to vaccination, Int. J. Cancer 141 (2017) 1576-1584.

[46] World Health Organization, ed, WHO Guidelines for Screening and Treatment of Precancerous Lesions for Cervical Cancer Prevention, World Health Organization, Geneva, 2013.

[47] J. Jeronimo, P. Bansil, M. Valdez, L.N. Kang, F.H. Zhao, Y.L. Qiao, et al., The influence of human papillomavirus genotypes on visual screening and diagnosis of cervical precancer and cancer, J. Low. Genit. Tract Dis. 19 (2015) 220-223.

[48] T. Dorjgochoo, A. Kallianpur, Y.T. Gao, H. Cai, G. Yang, H. Li, et al., Dietary and lifestyle predictors of age at natural menopause and reproductive span in the Shanghai Women's Health Study, Menopause 15 (2008) 924-933. 